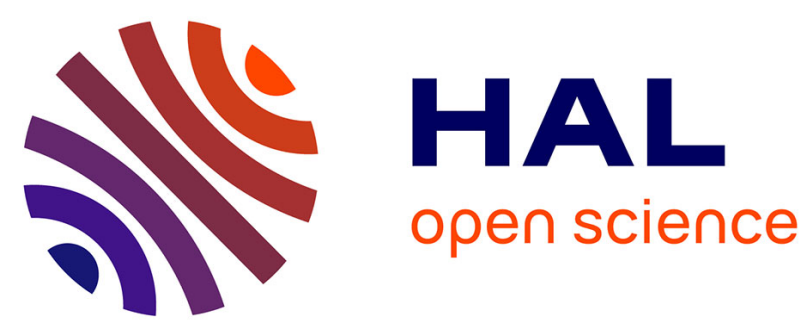

\title{
Charge électrique résiduelle sur des poudres à l'extinction d'un plasma poussiéreux
}

Lénaïc Couëdel, Abdelaziz Mezeghrane, Maxime Mikikian, Yves Tessier, Alexander A. Samarian, Cameron Cuthbert, Laifa Boufendi

\section{- To cite this version:}

Lénaïc Couëdel, Abdelaziz Mezeghrane, Maxime Mikikian, Yves Tessier, Alexander A. Samarian, et al.. Charge électrique résiduelle sur des poudres à l'extinction d'un plasma poussiéreux. Journal of Electrostatics, 2009, 67 (4), pp.625. 10.1016/j.elstat.2007.06.008 . hal-00391822

\section{HAL Id: hal-00391822 \\ https://hal.science/hal-00391822}

Submitted on 4 Jun 2009

HAL is a multi-disciplinary open access archive for the deposit and dissemination of scientific research documents, whether they are published or not. The documents may come from teaching and research institutions in France or abroad, or from public or private research centers.
L'archive ouverte pluridisciplinaire HAL, est destinée au dépôt et à la diffusion de documents scientifiques de niveau recherche, publiés ou non, émanant des établissements d'enseignement et de recherche français ou étrangers, des laboratoires publics ou privés. 


\title{
Charge électrique résiduelle sur des poudres à l'extinction d'un plasma poussiéreux
}

\author{
L. Couëdel ${ }^{\mathrm{a}, \mathrm{c}, *}$ A. Mezeghrane ${ }^{\mathrm{a}, \mathrm{b}}$ M. Mikikian ${ }^{\mathrm{a}}$ Y. Tessier ${ }^{\mathrm{a}}$ A.A. Samarian ${ }^{\mathrm{c}}$ C. Cuthbert ${ }^{\mathrm{c}}$ L. Boufendi ${ }^{\mathrm{a}}$ \\ ${ }^{a}$ GREMI (Groupe de Recherches sur l'Énergétique des Milieux Ionisés), UMR6606, CNRS-Université d'Orléans, 14 rue d'Issoudun, BP \\ 6744, 45067 Orléans cedex 2, France \\ ${ }^{\mathrm{b}} \mathrm{LPCQ}$, Université M. Mammeri, Tizi-ouzou, Algérie \\ ${ }^{\mathrm{c}}$ School of physics A28, University of Sydney, NSW 2006, Australie
}

\begin{abstract}
La charge électrique résiduelle des poudres à l'extinction d'un plasma poussiéreux a été mesurée dans une décharge radio-fréquence. La force de gravité a été compensée par l'application d'une force de thermophorèse de même intensité et de direction opposée. Des poudres chargées positivement ou négativement ainsi que des poudres non chargées ont été observées. Ces charges résiduelles peuvent se maintenir pendant plus de 1 minute après l'arrêt de la décharge. La charge électrique résiduelle moyenne pour des poudres d'environ $200 \mathrm{~nm}$ de rayon a été mesurée à environ -5e pour une pression de 1,2 mbar et - $3 e$ pour une pression de $0,4 \mathrm{mbar}$.

La charge électrique résiduelle sur les poudres étant reliée aux phénomènes de diffusion (ambipolaire et libre) des espèces chargées du plasma (ions et électrons), des mesures de densité électronique dans des plasmas post-décharges d'argon pur et d'argon/méthane (plasma poussiéreux) ont été effectuées afin de comprendre l'influence des poudres sur les mécanismes de perte des électrons. Ces mesures ont montré que la présence de poudres provoque d'abord une augmentation rapide de la densité électronique à l'arrêt du plasma suivie d'une décroissance accélérée.
\end{abstract}

Key words: plasma poussiéreux, charge électrique résiduelle, densité électronique PACS: 52.27.Lw

\section{Introduction}

Les plasmas poussiéreux (ou plasmas complexes) sont des gaz partiellement ionisés composés d'espèces neutres, d'ions, d'électrons et de poudres électriquement chargées. Ces poudres acquièrent une charge grâce aux interactions avec les ions et les électrons du plasma environnant [1-3]. En laboratoire, cette charge est négative à cause de la plus grande mobilité des électrons.

Des poudres dans un plasma ont été observées pour la première fois dans les années 1920 par Langmuir dans une décharge filamentaire [4]. Ces poudres venaient de la pulvérisation de la cathode de tungstène. Jusqu'à la fin des années 1980, les plasmas poussiéreux n'étaient pas étudiés activement mis à part quelques articles dans la communauté des astrophysiciens (voir par exemple [5,6]). En 1989, Selwyn découvrit des poudres dans un réacteur industriel de chez IBM en utilisant la diffusion de la lumière d'un laser [7]. Celles-ci s'étaient formées à l'intérieur du plasma

\footnotetext{
* lenaic.couedel@univ-orleans.fr
}

et étaient une source majeure de la contamination des substrats. La croissance de poudres dans les plasmas devint alors un problème majeur et une meilleure compréhension des processus physico-chimiques intervenant dans les plasmas poussiéreux fut nécessaire.

Dans les expériences de laboratoire, les poudres peuvent être injectées directement dans le plasma ou bien formées à l'intérieur de celui-ci. Les poudres injectées sont généralement de taille micrométrique et, à cause de leur masse, sont confinées près de l'électrode inférieure (gaine) où la force électrique est suffisante pour compenser la gravité. Des conditions de microgravité sont donc nécessaires pour pouvoir piéger les poussières dans tout l'espace inter-électrode [8]. On peut cependant obtenir en laboratoire des nuages denses de poudres submicrométriques remplissant tout le réacteur, soit en utilisant des gaz réactifs comme le silane $[9,10]$, le méthane ou l'acétylène [11], soit en pulvérisant une cible grâce aux ions du plasma $[12,13]$.

Le comportement des poudres à l'intérieur d'un plasma est déterminé par leur charge via leurs interactions avec 
les ions, les électrons, les autres poudres et les champs électriques environnants. De nombreux articles sont consacrés à l'étude des processus de charge des poussières aussi bien dans les plasmas de laboratoire [3,14] que dans les plasmas astrophysiques $[1,15]$. Néanmoins, il existe peu d'études sur la perte de charges à l'arrêt du plasma $[16,17]$. Ainsi, chaque nouvelle expérience sur les plasmas poussiéreux en phase post-décharge permet de mieux comprendre les phénomènes physiques des plasmas complexes. Dans cette article nous présentons des résultats sur la mesure de la décroissance électronique durant cette phase ainsi que des résultats de mesure de charges électriques résiduelles sur les poudres après l'extinction du plasma. La nature et la valeur de cette charge résiduelle est en effet très importante. Elle peut par exemple être source de problèmes sur les futurs dispositifs électroniques à un électron où une charge résiduelle attachée aux nanocristaux déposés serait à l'origine de dysfonctionnements. Elle peut en revanche être utile pour la décontamination des réacteurs plasmas industriels grâce à l'utilisation de champs électriques appropriés. Cette propriété peut aussi être utilisée dans les réacteurs pour la fusion nucléaire (comme ITER) en facilitant l'élimination des grains de poussières créés par l'érosion des parois exposées au plasma. La charge électrique résiduelle étant reliée aux phénomènes de diffusion des espèces chargées (ions et électrons) dans la phase post-décharge, il est nécessaire de mesurer et comprendre l'influence des poudres sur ceux-ci.

\section{Dispositifs expérimentaux}

\subsection{Le réacteur PKE-Nefedov (Plasma Kristall Experiment)}

Ce réacteur a été conçu à l'origine pour des expériences en microgravité [8]. C'est une décharge radio-fréquence (RF) excitée en mode push-pull. Elle est constituée de deux électrodes parallèles de $4 \mathrm{~cm}$ de diamètre séparées de $3 \mathrm{~cm}$. La puissance injectée varie entre $0 \mathrm{~W}$ et $4 \mathrm{~W}$. Les poudres sont formées dans un plasma d'argon (0,2 - 2 mbar $)$ par pulvérisation d'une couche de polymère déposée sur les électrodes et composée de poussières injectées précédemment (3,4 $4 \mathrm{~m}$, mélamine formaldéhyde). Une description détaillée de cette expérience ainsi que les précédents résultats sont donnés dans les références $[8,18,13]$.

Pour l'étude des charges résiduelles, l'électrode supérieure a été refroidie (Fig. 1) créant ainsi une force de thermophorèse compensant la gravité [19] quand le plasma est éteint. Pour mesurer la charge résiduelle des poudres, une tension sinusoïdale, créée par un générateur de fonctions, d'amplitude $\pm 30 \mathrm{~V}$ et de fréquence $1 \mathrm{~Hz}$ a été appliquée à l'électrode inférieure. Le champ électrique sinusoïdal basse fréquence induit $\boldsymbol{E}(\boldsymbol{r}, t)$ entraîne l'oscillation des poudres si celles-ci gardent une charge électrique résiduelle.

Une nappe laser de faible épaisseur éclaire le nuage de poudres et la lumière diffusée est enregistrée à $90^{\circ}$ par des caméras CCD à 25 images par seconde. Les signaux vidéo sont transférés sur un ordinateur grâce à une carte d'acquisition sur une échelle de gris de 8 bits de profondeur et une résolution de $560 \times 700$ pixels. Afin d'éviter les effets de bord, le domaine analysé pour la mesure des charges résiduelles est restreint à une région de $8.53 \times 5.50 \mathrm{~mm}^{2}$ centrée au milieu de l'espace inter-électrode. Par superposition des images vidéo, les trajectoires des poudres ont été obtenues. Les coordonnées des grains ont été relevées toutes les 3 images et l'amplitude des oscillations a été obtenue grâce à ces mesures.

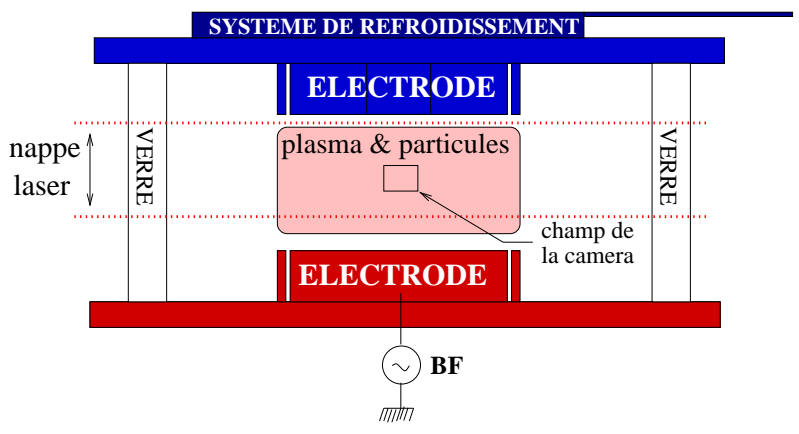

Fig. 1. Schéma du dispositif expérimental

En raison de sa conception, ce réacteur ne permet pas de mesurer aisément les paramètres du plasma. Par conséquent, il a été necessaire d'utiliser un autre réacteur pour faire les mesures de densité électronique durant la phase post-décharge. Pour cela, nous avons utilisé un réacteur où l'on peut faire croître des poudres à l'aide de gaz réactifs. Afin d'obtenir des poudres de composition chimique proche de celles formées par pulvérisation dans le réacteur PKE (poudres de matière carbonée), nous avons utilisé un mélange argon-méthane.

\subsection{Le réacteur dédié aux gaz réactifs}

La décharge RF est créée dans une boite cylindrique (réliée à la masse) de $13 \mathrm{~cm}$ de diamètre. L'espace interélectrode est de $3,3 \mathrm{~cm}$. Ce système est enfermé dans une enceinte sous vide. L'électrode supérieure est reliée au générateur radiofréquence à $13,56 \mathrm{MHz}$ par une boite d'accord incluant une capacité de blocage. Cette électrode a la forme d'une pomme de douche afin d'assurer une distribution homogène du gaz dans la zone du plasma. Une description détaillée de ce dispositif expérimental a été donné dans d'autres articles [20-22].

Les expériences sont effectuées en argon pur et en mélange argon/méthane. La pression de gaz ést de 1,1 mbar et la puissance injectée est de $20 \mathrm{~W}$. Le débit d'argon est réglé à environ $40 \mathrm{sccm}$ et celui de méthane à approximativement $0,8 \mathrm{sccm}$.

Les mesures de densité électronique sont réalisées en utilisant la technique de la cavité résonante micro-onde. 
Pour cela, deux antennes sont fixées au fond de la boîte à décharge en vis-à-vis. La première antenne est reliée à un générateur micro-onde dont la fréquence varie entre $100 \mathrm{kHz}$ et $4 \mathrm{GHz}$. La deuxième antenne receuille le signal. En cherchant, les modes de résonance à vide et en plasma, il est alors possible de remonter à la densité électronique [23-25].

\section{Forces agissant sur les poudres}

Les poudres immergées dans un plasma sont soumises à plusieurs forces qui soit les confinent dans ce plasma soit les éjectent (vers les parois ou les ports de pompage) [25]: les forces électrostatiques, la gravité, la force de thermophorèse, la force de friction des ions et la force de friction des neutres.

Lorsque la décharge est éteinte, les forces continuant d'agir sur les poudres sont la force de gravité $F_{g}$, la force de thermophorèse $F_{T}$ et la friction avec les neutres $F_{d n}$. Afin de vérifier si les poussières gardent une charge électrique résiduelle à l'extinction du plasma, un champ électrique sinusoïdal basse fréquence est créé par la polarisation de l'électrode inférieure engendrant le cas échéant une force électrique $F_{E}$ sur les poudres. Dans cette expérience, la gravité est compensée par la force de thermophorèse et les frottements des neutres amortissent les oscillations induites par la force électrique.

Le poids de la particule est donné par:

$$
\boldsymbol{F}_{\boldsymbol{g}}=(4 / 3) \pi r_{d}^{3} \cdot \rho \boldsymbol{g}
$$

où $\boldsymbol{g}$ est l'accélération gravitationnelle, $r_{d}$ le rayon de la poudre, et $\rho$ sa masse volumique.

La force de friction due aux neutres est donnée par [26]:

$$
\boldsymbol{F}_{\boldsymbol{d n}}=-(8 / 3) \sqrt{2 \pi} r_{d}^{2} m_{n} n_{n} v_{T n}\left(1+\alpha_{a c} \frac{\pi}{8}\right)\left(\mathbf{v}_{\mathbf{d}}-\mathbf{v}_{\mathbf{n}}\right)
$$

où $m_{n}$ est la masse des atomes neutres du gaz, $n_{n}$ leur densité, $v_{T n}=\sqrt{8 k_{B} T_{n} / \pi m_{n}}$ leur vitesse thermique avec $T_{n}=T$ la température du gaz neutre, $k_{B}$ la constante de Boltzmann, $\alpha_{a c}$ le coefficient d'accommodation, $\mathbf{v}_{\mathbf{d}}$ la vitesse des poudres et $\mathbf{v}_{\mathbf{n}}$ la vitesse moyenne des atomes du gaz neutre. Dans notre expérience, $\mathbf{v}_{\mathbf{n}}$ est nul car il n'y a pas de flux de gaz.

La force électrique s'écrit:

$$
\boldsymbol{F}_{\boldsymbol{E}}(\boldsymbol{r}, t)=Q_{d} \boldsymbol{E}(\boldsymbol{r}, t)
$$

où $Q_{d}$ est la charge électrique de la poudre et $\boldsymbol{E}(\boldsymbol{r}, t)$ le champ électrique entre les électrodes après l'extinction du plasma. Durant la phase post-décharge, la densité du "plasma" devient rapidement très faible et $\boldsymbol{E}(\boldsymbol{r}, t)$ peut être approximé par le champ au dessus d'un disque chargé. En prenant en compte la taille de la zone étudiée, celle des électrodes et le fait que l'on étudie une région proche du centre du réacteur, seule la composante verticale du champ électrique $\boldsymbol{E}(\boldsymbol{r}, t) \approx E_{z}(z, t) \vec{e}_{z} \equiv E(z, t) \vec{e}_{z}$ intervient.
L'expression de la force de thermophorèse doit quant à elle être choisie soigneusement. En effet, elle dépend fortement du nombre de Knudsen $K n=l / r_{d}$ [27] où $l$ est le libre parcours moyen des espèces du gaz. Dans notre expérience, nous travaillons avec une pression aux alentours de 1 mbar. Dans un article précédent [13], la taille des poudres formées a été mesurée entre $200 \mathrm{~nm}$ et $800 \mathrm{~nm}$. Cela donne, en utilisant les résultats de Varney [28] pour la section efficace atome-atome, un nombre de Knudsen $250<K n<1000$. Par conséquent, nous sommes en régime moléculaire où une poudre peut être considérée comme une très grosse molécule. Beaucoup de théories ont été développées [29-32,27] et utilisées [19,33,27] pour la thermophorèse en régime moléculaire. L'équation la plus souvent employée est celle de Waldmann [29] qui a été vérifiée expérimentalement [34,35]:

$$
\boldsymbol{F}_{\boldsymbol{T}}=-(32 / 15) r_{d}^{2}\left(k_{t r} / v_{T n}\right) \boldsymbol{\nabla} T
$$

où $\nabla T$ est le gradient de température dans le gaz et $k_{t r}$ la partie translationnelle de la conductivité thermique donnée pour un gaz monoatomique par [36]:

$$
k_{t r}=\left(\left(15 k_{B}\right) /\left(4 m_{n}\right)\right) \mu_{r e f}\left(T / T_{r e f}\right)^{\nu}
$$

où $\mu_{\text {ref }}$ est la viscosité à la température de référence $T_{\text {ref }}=273 \mathrm{~K}$. L'exposant $\nu$ est obtenu par ajustement des données expérimentales aux alentours de la température de référence. Pour l'argon, $\mu_{\text {ref }}=2,117 \cdot 10^{-5} \mathrm{~Pa} \cdot \mathrm{s}$ et $\nu=0,81[36]$.

Un autre effet important qui doit être pris en compte pour estimer la force de thermophorèse est l'influence du volume fini de gaz. Si la pression est suffisamment basse, le libre parcours moyen du gaz peut être comparable aux longueurs caractéristiques du dispositif expérimental et le gaz ne peut plus être considéré comme un milieu continu. Sous de telles conditions, un nombre de Knudsen supplémentaire doit être ajouter [27] $K n_{L}=l / L$ où $L$ est la longueur caractéristique du réacteur. Dans cette expérience, l'espacement entre les électrodes est de $L=$ $3 \mathrm{~cm}$ donnant $K n_{L} \sim 5 \cdot 10^{-3}$ indiquant que l'on peut bien considérer notre gaz comme un milieu continu.

Le gradient de température entre les deux électrodes a été calculé en utilisant le logiciel FEMLAB ${ }^{\circledR}$ (analyse de l'état à l'équilibre des transferts de chaleur avec convection et conduction en utilisant des conditions aux limites de flux de chaleur, de températures, éléments de Lagrange quadratiques). La température des électrodes a été mesurée grâce à un thermocouple et utilisée comme conditions aux limites pour le problème. Le résultat obtenu a montré que la composante verticale du gradient de température est constante au centre du réacteur. Sa valeur est d'environ $2 \mathrm{~K} / \mathrm{cm}$ pour notre expérience. Ce gradient a aussi une faible composante horizontale provoquant une faible dérive horizontale des poudres. Cette dérive nous permet néanmoins de reconstruire plus facilement les trajectoires de ces dernières et facilite par conséquent la mesure des charges électriques résiduelles. 


\section{Résultats expérimentaux}

\subsection{Charges électriques résiduelles sur les poudres}

Les charges résiduelles sont mesurées en utilsant le protocole expérimental suivant. Le réacteur est d'abord pompé jusqu'à la plus faible pression possible (pression de base $\sim 2 \cdot 10^{-6}$ mbar ) et le système de refroidissement est mis en marche. Ensuite, de l'argon est injecté jusqu'à la pression désirée et la décharge allumée. Des poudres se forment et grossissent dans le plasma et s'organisent suivant des structures comme le "void" [18]. Enfin, le plasma est éteint et une tension sinusoïdale basse fréquence appliquée à l'électrode inférieure.

En post-décharge, la dynamique des poudres est déterminée par le gradient de température et le champ électrique d'excitation. La Fig. 2 montre une superposition d'images prises après l'extinction du plasma. On peut observer deux différents types de mouvements: des poudres dérivent vers le haut, vers le bas ou horizontalement et elles oscillent à cause de la force électrique appliquée. Il est évident que la force de thermophorèse agit sur toutes les poussières alors que la force électrique n'agit que sur celles qui conservent une charge électrique en phase post-décharge. Ainsi la présence de poudres oscillantes (voir Fig. 2) indique clairement que celles-ci gardent une charge résiduelle. Des poudres oscillant en opposition de phase ainsi que d'autres n'oscillant pas ont aussi été observées montrant ainsi la présence de poudres chargées positivement ou négativement ainsi que de poudres non-chargées.

Afin d'observer des charges résiduelles, la décharge doit être éteinte rapidement. En effet, si on diminue la puissance très progressivement jusqu'à ce que le plasma s'éteigne tout seul, il n'y a pas d'oscillations détectables. Ce phénomène sera le sujet d' études ultérieures. Les charges résiduelles sur les poudres ont un long temps de relaxation et ne dépendent pas du moment où l'on applique le champ électrique d'excitation. Des poudres oscillantes sont en effet observées pendant plus d'une minute après l'extinction du plasma, que le générateur de fonctions soit mis en marche avant ou après cette extinction.

Comme on peut le voir sur la Fig. 2, certaines poudres tombent après l'extinction de la décharge. Celles-ci sont trop lourdes pour être maintenues par la force de thermophorèse. D'autres dérivent horizontalement indiquant que leur poids est compensé par la force de thermophorèse. Ce sont ces particules qui sont utilisées pour la mesure des charges résiduelles. La Fig. 2 montre également que l'utilisation d'une caméra ayant un large champ de vue n'est pas adaptée à la mesure car les effets de bord ne peuvent être négligés. Ainsi une caméra ayant un champ réduit au centre de la décharge est employée. Une superposition d'images issues de cette caméra est présentée sur la Fig. 3. Celle-ci nous donne une empreinte claire des oscillations des poudres et peut donc être utilisée pour la reconstruction des trajectoires (Fig. 4).

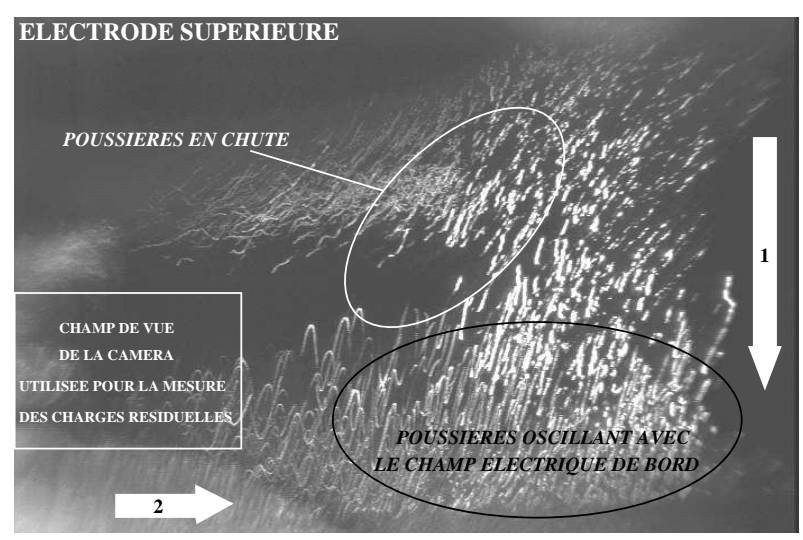

Fig. 2. Superposition d'images prises avec la caméra ayant un champ de vue large. Les flèches 1 et 2 représentent respectivement les composantes verticales et horizontales du gradient de température. Les effets de bords ainsi que des poudres tombantes peuvent être observés.

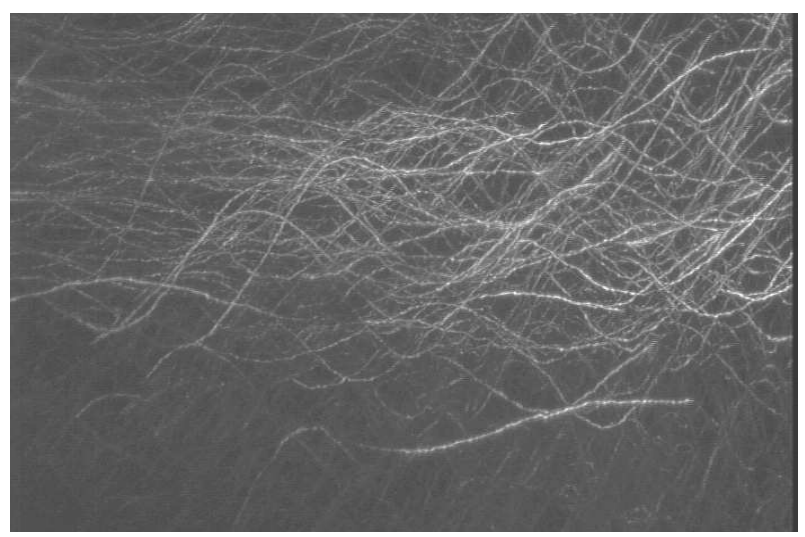

Fig. 3. Superposition d'images vidéo quelques secondes après l'arrêt $\mathrm{du}$ plasma. Les oscillations des poudres sont clairement observables. Le gradient de température ayant une légère composante horizontale, les oscillations sont caractérisées par un déplacement des poudres vers la gauche de l'image

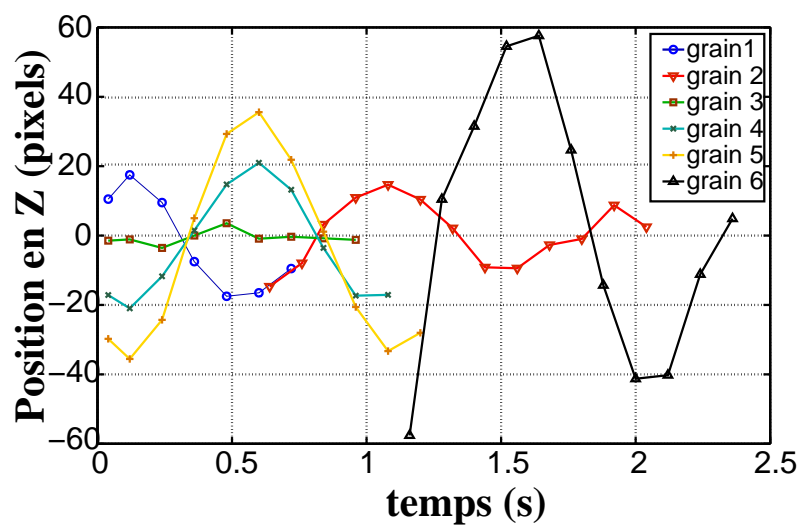

Fig. 4. Oscillations de 6 poudres, 10 secondes après l'extinction du plasma (1 pixel $\sim 11 \mu \mathrm{m}$ ). Des poudres n'oscillant pas ainsi que des oscillations en opposition de phase sont observées. 


\subsubsection{Taille et masse des poudres levitant en phase} post-décharge

La mesure des charges résiduelles est fortement liée à la détermination de la taille (masse) des poudres. Par conséquent, il est nécessaire d'évaluer ces paramètres. Les poussières qui lévitent à une hauteur constante après l'extinction du plasma sont celles dont le poids est compensé par la force de thermophorèse. Par conséquent, leur rayon est obtenue en utilisant les Eqs. (1) et (4):

$$
r_{d}=-\frac{8}{5 \pi \rho g} \frac{k_{t r}}{v_{t h}} \nabla T
$$

Ces poudres sont supposées sphériques et principalement constituées de carbone (pulvérisation d'un polymère carboné) [13]. Ainsi la masse $m_{d}$ est donnée en utilisant Eq.(6) par:

$$
m_{d}=(4 / 3) \pi r_{d}^{3} \cdot \rho
$$

où $\rho$ est la masse volumique du graphite. Dans nos conditions expérimentales, le rayon des particules est estimé à $r_{d} \simeq 190 \mathrm{~nm}$ et leur masse à $m_{d} \simeq 6,5 \cdot 10^{-17} \mathrm{~kg}$.

\subsubsection{Mesure de la charge électrique résiduelle}

A partir de la mesure de l'amplitude des oscillations des poudres, on peut obtenir leurs charges résiduelles. Comme le poids des poussières est compensé par la force de thermophorèse, l'équation du mouvement pour une poudre peut s'écrire en négligeant les interaction avec ses voisines:

$$
m_{d} \ddot{z}=F_{E}(z, t)+F_{n d}(\dot{z})
$$

En prenant $E(t)=E_{0}\left(z_{\text {mean }}\right) \cos (\omega t)$ (l'amplitude du champ électrique $E_{0}$ est celle à la hauteur moyenne de lévitation de la poudre $z_{\text {mean }}$ ) et en utilisant les Eq.(3), Eq.(2) et Eq.(8), l'amplitude d'oscillation $b$ de la poudre peut être obtenue [37]:

$$
b\left(\omega, Q_{d}, E_{0}\right)=\frac{Q_{d} E_{0}\left(z_{\text {mean }}\right)}{m_{d} \omega \sqrt{\omega^{2}+4 \gamma^{2} / m_{d}^{2}}}
$$

où $\gamma=(4 / 3) \sqrt{2 \pi} r_{d}^{2} m_{n} n_{n} v_{T n}\left(1+\alpha_{a c}(\pi / 8)\right)$ est le coefficient d'amortissement et $\omega=2 \pi f$ où $f$ est la fréquence imposée par le générateur de fonctions. L'Eq. (9) peut être facilement inversée pour obtenir la charge résiduelle $Q_{d_{r e s}}$ de la poudre:

$$
Q_{d_{\text {res }}}=\frac{m_{d} b\left(\omega, Q_{d}, E_{0}\left(z_{\text {mean }}\right)\right) \omega \sqrt{\omega^{2}+4 \gamma^{2} / m_{d}^{2}}}{E_{0}\left(z_{\text {mean }}\right)}
$$

Des amplitudes d'oscillations pouvant atteindre $1.1 \mathrm{~mm}$ ont été mesurées et des charges de $-12 e$ à $+2 e$ ont été déterminées où $e$ est la charge élémentaire. En reconstruisant les oscillations de plusieurs poudres et en mesurant leurs amplitudes, nous avons obtenu les distributions de charges résiduelles (Fig. 5). On remarque que ces distributions peuvent être ajustées par des gaussiennes. Il a de plus été trouvé que les poudres gardent une charge résiduelle moyenne plus importante à haute pression (Fig. $5)$. L'incertitude sur les mesures est d'environ $2 e$.
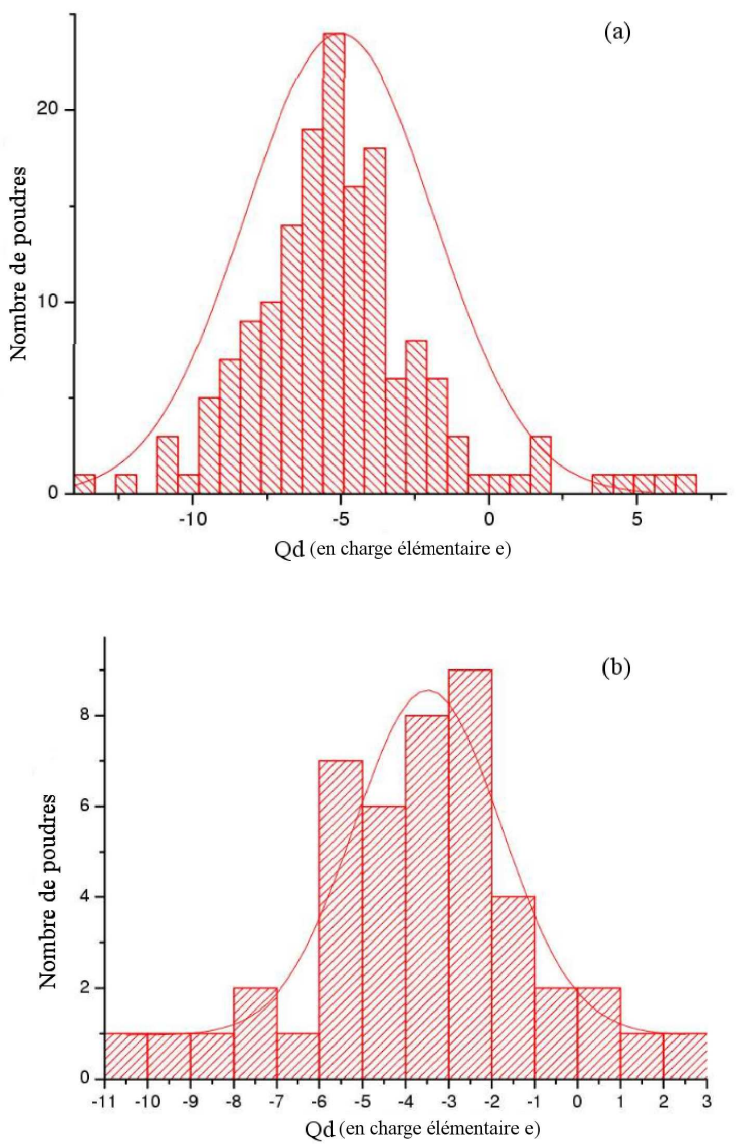

Fig. 5. Distribution de charges pour une pression de (a) 1,2 mbar, (b) 0,4 mbar

\subsection{Mesure de la densité électronique en plasma post-décharge}

Les mesures de densité électronique sont effectuées en utilisant le protocole expérimental suivant. L'enceinte est d'abord pompé jusqu'à obtenir le meilleur vide possible $\left(\sim 10^{-6} \mathrm{mbar}\right)$. Les gaz sont ensuite injectés aux débits voulus et la pression ajustée à 1, 1 mbar.

Les fréquences de résonance à vide (sans plasma) de la cavité résonante ont alors été déterminées et nous avons décidé d'utiliser le mode de résonance à $2720 \mathrm{MHz}$ (mode TM 110) qui est l'un des plus sensibles.

Pour un mélange et une durée d'allumage donnés, les résultats expérimentaux sont reproductibles. Nous avons donc mesuré la réponse de la cavité pour chaque fréquence autour de la fréquence à vide par pas de $1 M h z$ avec une résolution temporelle de 500 ns. Nous avons ensuite reconstruit les courbes de résonance à chaque instant $t$ de la phase post-décharge.

Pour les mesures en argon pur, la durée d'allumage est de $15 s$ avec un temps d'attente de $30 s$ entre deux plasmas nécessaire pour revenir aux conditions initiales. Pour le mélange argon-méthane, le temps d'allumage était de $43 \mathrm{~s}$ 
avec un temps d'attente de $85 s$ entre deux plasmas. La puissance injectée dans le plasma est de $20 \mathrm{~W}$ dans les deux cas.

En mesurant l'écart entre la fréquence de résonance à vide et la fréquence de résonance aux différents instants de la phase post-décharge, les courbes de densité électronique peuvent être reconstruites grâce à la formule [25]:

$$
n_{e}(t)=\frac{2 m_{e} \epsilon_{0}(2 \pi f(t))^{2}}{e^{2}} \frac{\Delta f(t)}{f_{0}}
$$

où $f_{0}$ est la fréquence de résonance à vide, $f(t)$ la fréquence de résonance à l'instant $t, \Delta f(t)=f(t)-f_{0}$. Les résultats obtenus sont présentés sur la Fig. 6 .

On remarque que la densité électronique en phase plasma
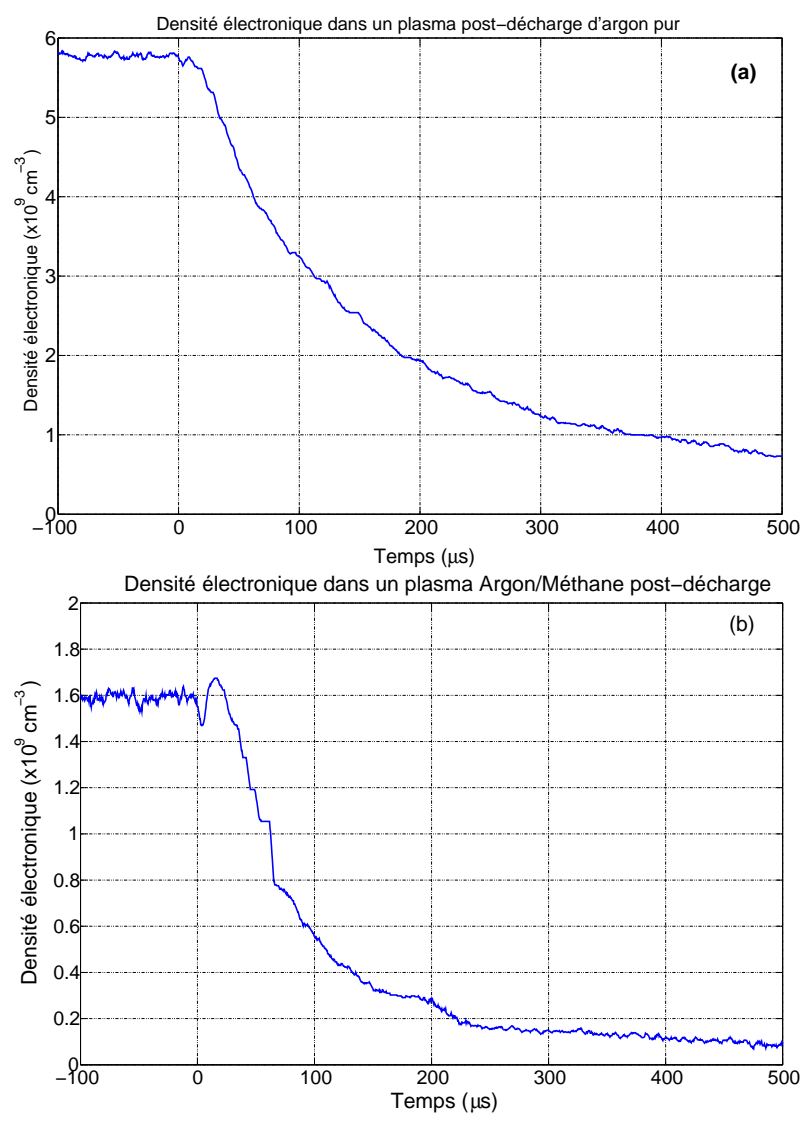

Fig. 6. (a) Evolution de la densité électronique dans un plasma d'argon pur. (b) Evolution de la densité électronique dans un plasma poussiéreux de mélange argon/méthane. Dans les deux cas, on arrête le décharge a $\mathrm{t}=0$.

$(t<0)$ est plus faible en mélange argon/méthane (Fig. 6b) qu'en argon pur (Fig. 6a). Cela est dû à l'attachement électronique sur les poudres qui se forment dans la phase plasma.

Nous avons mesuré les temps caractéristiques de la perte d'électrons à l'arrêt de la décharge et $500 \mu s$ après (on suppose $n_{e}(t) \propto \exp \left(-t / \tau_{L}(t)\right)$ où $\tau_{L}(t)$ est le temps de diffusion). Dans le cas de l'argon pur, on a obtenu $\tau_{0} \simeq 125 \mu \mathrm{s}$ et $\tau_{500} \simeq 480 \mu \mathrm{s}$. Pour le mélange argon/méthane, on a obtenu $\tau_{0} \simeq 75 \mu \mathrm{s}$ et $\tau_{500} \simeq 300 \mu \mathrm{s}$. La perte d'électrons en phase post-décharge $(t>0)$ est donc plus rapide en présence de poudres. Cette accélération de la perte des électrons est due à la recombinaison des ions et électrons à la surface des poudres en plus de la diffusion ambipolaire $[16,17]$. De plus, on remarque que la diminution de la densité électronique n'est pas immédiate en présence de poudres mais est d'abord précédée par une légère augmentation de celle-ci formant un pic d'une durée $t_{p i c} \simeq 35 \mu \mathrm{s}$. Un tel pic mais d'une durée beaucoup plus longue $\left(\sim 10 \cdot t_{\text {pic }}\right)$ a déjà été observé par d'autres équipes dans les premiers instants de la phase post-décharge [38,39]. L'explication avancée est une réémission par les poudres des électrons capturés précédemment. Cependant, cette réémission pourrait être dépendante de la composition chimique des poudres. Par conséquent des expériences dans une autre chimie (argonsilane) sont actuellement en cours.

\section{Discussion et conclusion}

La charge électrique des poudres en phase plasma est donnée dans le cas d'une poudre isolée par [26]:

$$
Q_{d}=\left(4 \pi \epsilon_{0} r_{d} k_{B} T_{e} / e\right) \ln \left(\frac{n_{i}}{n_{e}} \cdot\left(\frac{m_{e} T_{e}}{m_{i} T_{i}}\right)^{1 / 2}\right)
$$

où $T_{e} \sim 3 \mathrm{eV}$ est la température électronique, $T_{i} \sim 0,03 \mathrm{eV}$ est la température ionique, $n_{e(i)}$ est la densité électronique (ionique) et $m_{e(i)}$ est la masse d'un électron (ion). Dans les conditions expérimentales pour la mesure des charges résiduelles, la charge en phase plasma est $Q_{d 0} \sim-950 e$. Cette expérience nous a permis de montrer que la charge résiduelle gardée par les poudres est $Q_{d_{\text {res }}} \sim 0,005 \cdot Q_{d 0}$. Une explication possible pour l'existence de cette charge résiduelle est une influence des poudres lors du processus de perte des charges (ions et électrons) du plasma et en particulier un arrêt anticipé de la diffusion ambipolaire dû au volume de charge non négligeable des poudres (ions en excès par rapport aux électrons) [17]. En effet, les mesures de la densité électronique dans la phase post-décharge dans un cas sans poudres et un cas avec poudres ont révélé l'influence non négligeable de celles-ci. Elles contribuent d'abord à une légère augmentation de la densité électronique avant d'accélèrer la perte des électrons dans la phase post-décharge. Des études similaires [38,39] ont montré des phénomènes semblables aux premiers instants de la diffusion. Une explication avancée est la réémission par les poudres des électrons capturés précédemment. Des expériences complémentaires dans d'autres chimie (argonsilane) sont actuellement en cours afin de vérifier si ce phénomène est dépendant ou non de la composition chimique des poudres.

Le modèle [17] n'est pas complet car il ne permet pas d'expliquer la présence de poudres chargées positivement ainsi que la dépendance aux conditions initiales du plasma. Un modèle prenant en compte différents phénomènes physiques (réchauffement des électrons, émission d'électrons [38,39], chimie de la phase postdécharge) dans les plasmas en extinction doit donc être 
développé afin de mieux comprendre la phase post-décharge des plasma poussiéreux.

\section{Remerciements}

Les auteurs souhaitent remercier S. Dozias et B. Dumax pour le support électronique et J. Mathias pour le support optique. Le réacteur PKE-Nevedov a été mis à disposition par le Max-Planck-Institute for Extraterrestrial Physics, Allemagne, grâce au financement de la DLR/BMBF avec la bourse No. 50WM9852. Ce travail est supporté par le CNES avec le contrat No. 793/2000/CNES/8344. Ce travail est aussi partiellement supporté par le programme de recherche intégré franco-australien du ministère français des affaires étrangères (FAST) et les liens scientifiques internationaux établis grâce au rapport sur l'innovation du gouvernement australien supporté par ce gouvernement et le projet de recherche et développement de l'Université de Sydney.

\section{References}

[1] O. Havnes, T. K. Aanesen, F. Melandsø, On dust charges and plasma potentials in a dusty plasma with dust size distribution, J. Geophys. Res. 95 (A5) (1990) 6581-6585.

[2] T. Matsoukas, M. Russell, Particle charging in low-pressure plasmas, J. Appl. Phys. 77 (1995) 4285.

[3] C. Arnas, M. Mikikian, F. Doveil, High negative charge of a dust particle in a hot cathode discharge, Phys. Rev. E 60 (1999) 7420.

[4] I. Langmuir, C. G. Found, A. F. Dittmer, A new type of electric discharge: the streamer discharge, Science LX (1557) (1924) 392.

[5] O. Havnes, G. E. Morfill, Effects of electrostatic forces on the vertical structure of planetary rings, Adv. Space Res. 4 (9) (1984) $85-90$.

[6] O. Havnes, Charges on dust particles, Adv. Space Res. 4 (1984) $75-83$.

[7] G. S. Selwyn, J. Singh, R. S. Bennett, In situ laser diagnostic studies of plasma-generated particulate contamination, J. Vac. Sci. Technol. A 7 (4) (1989) 2758-2765.

[8] A. P. Nefedov, G. E. Morfill, V. E. Fortov, H. M. Thomas, et al., PKE-Nefedov: plasma crystal experiments on the international space station, New J. Phys. 5 (2003) 33.

[9] A. Bouchoule, L. Boufendi, Particle formation and dusty plasma behaviour in argon-silane rf discharge, Plasma Sources Sci. Technol. 2 (1993) 204-213.

[10] M. Cavarroc, M. C. Jouanny, K. Radouane, M. Mikikian, L. Boufendi, Self-excited instability occurring during the nanoparticle formation in an $\mathrm{Ar} / \mathrm{SiH}_{4}$ low pressure radio frequency plasma, J. Appl. Phys. 99 (2006) 064301.

[11] S. Hong, J. Berndt, J. Winter, Growth precursors and dynamics of dust particle formation in the $\mathrm{Ar} / \mathrm{CH}_{4}$ and $\mathrm{Ar} / \mathrm{C}_{2} \mathrm{H}_{2}$ plasmas, Plasma Sources Sci. Technol. 12 (2003) 46-52.

[12] D. Samsonov, J. Goree, Particle growth in a sputerring discharge, J. Vac. Sci. Technol. A 17 (5) (1999) 2835.

[13] M. Mikikian, L. Boufendi, A. Bouchoule, H. M. Thomas, G. E. Morfill, A. P. Nefedov, V. E. Fortov, the PKE-Nefedov team, Formation and behaviour of dust particle clouds in a radiofrequency discharge: results in the laboratory and under microgravity conditions, New J. Phys. 5 (2003) 19.

[14] A. A. Samarian, S. V. Vladimirov, Charge of a macroscopic particle in a plasma sheath, Phys. Rev. E 67 (2003) 066404.

[15] J. Pavlu, I. Richterova, J. Safrankova, Z. Nemecek, Impact of surface properties on the dust grain charging, Adv. Space Res. In press (2005) Corrected proof.
[16] A. Ivlev, M. Kretschmer, M. Zuzic, G. E. Morfill, H. Rothermel, H. Thomas, V. E. Fortov, V. I. Molotkov, A. P. Nefedov, A. Lipaev, O. F. Petrov, Y. Baturin, A. I. Ivanov, J. Goree, Decharging of complex plasmas: First kinetic observations, Phys. Rev. Lett. 90 (2003) 055003.

[17] L. Couëdel, M. Mikikian, L. Boufendi, A. A. Samarian, Residual dust charge in discharge afterglow, Phys. Rev. E 74 (2) (2006) 026403.

[18] M. Mikikian, L. Boufendi, Experimental investigations of void dynamics in a dusty discharge, Phys. Plasmas 11 (8) (2004) 3733.

[19] H. Rothermel, T. Hagl, G. E. Morfill, M. H. Thoma, H. Thomas, Gravity compensation in complex plasmas by application of a temperature gradient, Phys. Rev. Lett. 89 (2002) 175001.

[20] A. Bouchoule, A. Plain, L. Boufendi, J. P. Blondeau, C. Laure, Particle generation and behavior in a silane-argon low-pressure discharge under continuous or pulsed radio-frequency excitation, J. Appl. Phys. 70 (1991) 1991.

[21] L. Boufendi, A. Plain, J. P. Blondeau, A. Bouchoule, C. Laure, M. Toogood, Measurements of particle size kinetics from nanometer to micrometer scale in a low-pressure argon-silane radio-frequency discharge, Appl. Phys. Lett 60 (1992) 169.

[22] L. Boufendi, A. Bouchoule, R. K. Porteous, J. P. Blondeau, A. Plain, C. Laure, Particle-particle interactions in dusty plasmas, J. Appl. Phys. 73 (1993) 2160.

[23] B. Agdur, B. Enander, Resonances of a microwave cavity partially filled with a plasma, J. Appl. Phys. 33 (2) (1962) 575581.

[24] W. W. Stoffels, E. Stoffels, G. M. W. Kroesen, F. J. de Hoog, Electron density fluctuations in a dusty $\mathrm{Ar} / \mathrm{SiH}_{4} \mathrm{rf}$ discharge, J. Appl. Phys. 78 (1995) 4867-4872.

[25] A. Bouchoule, Dusty Plasmas: Physics, Chemistry and Technological impacts in Plasma Processing, Wiley, New York, 1999.

[26] P. K. Shukla, A. A. Mamun, Introduction to dusty plasma, IOP Publishing, 2002.

[27] F. Zheng, Thermophoresis of spherical and non-spherical particles: a review of theories and experiments, Adv. Colloid \& Interface Sci. 97 (2002) 255-278.

[28] R. N. Varney, Drift velocities of ions in krypton and xenon, Phys. Rev. 88 (2) (1952) 362-364.

[29] L. Waldmann, Uber die kraft eines inhomogenen gases auf kleine suspendierte kugeln, Z. Naturforsch 14a (1959) 589-599.

[30] Z. Li, H. Wang, Thermophoretic force and velocity of nanoparticles in the free molecule regime, Phys. Rev. E 70 (2004) 021205.

[31] I. Goldhirsch, D. Ronis, Theory of thermophoresis. i. general considerations and mode-coupling analysis, Phys Rev. A 27 (3) (1983) 1616.

[32] I. Goldhirsch, D. Ronis, Theory of thermophoresis. ii. low density behaviour, Phys. Rev. A 27 (3) (1983) 1635.

[33] K. D. Bleecker, A. Bogaerts, Role of the thermophoretic force on the transport of nanoparticles in dusty silane plasmas, Phys. Rev. E 71 (2005) 066405.

[34] W. Li, E. J. Davis, Measurement of the thermophoretic force by electrodynamic levitation: Microspheres in air, J. Aerosol Sci. 26 (1995) 1063-1083.

[35] W. Li, E. J. Davis, The effects of gas and particle properties on thermophoresis, J. Aerosol Sci. 26 (1995) 1085-1099.

[36] M. A. Gallis, D. J. Rader, J. R. Torczynski, Thermophoresis in rarefied gas flows, Aerosol Sci. \& Technol. 36 (2002) 1099-1117.

[37] L. Landau, E. Lifpchitz, Physique théorique: tome 1, Mir, Moscou, 1986.

[38] J. Berndt, E. Kovacevic, V. Selenin, I. Stefanovic, J. Winter, Anomalous behaviour of the electron density in a pulsed complex plasma, Plasma Sources Sci. Technol. 15 (2006) 18-22.

[39] I. Stefanovic, J. Berndt, D. Maric, V. Samara, M. RadmilovicRadjenovic, Z. L. Petrovic, E. Kovacevic, J. Winter, Secondary electron emission of carbonaceous dust particles, Phys. Rev. E 74 (2006) 026406. 